\title{
Estimating slope from raster data: a test of eight different algorithms in flat, undulating and steep terrain
}

\author{
J. Tang \& P. Pilesjö \\ Department of Physical Geography and Ecosystem Analysis, \\ Lund University, Sweden
}

\begin{abstract}
Eight frequently used slope algorithms based on a DEM (Digital Elevation Model) have been compared in flat, gently sloping/undulating, and steep terrain in order to investigate differences in estimated results. The matter of scale/resolution has not been considered, and the focus has not been on comparing the estimates with "ground truth" data but on comparisons between the different algorithms. Pair-wise statistical tests have been carried out to detect significant differences between the methods in general, and also between different terrains. In this way, we make explanations and recommendations regarding these differences and "best practice" depending on data/terrain.

Keywords: DEM, slope, algorithm, terrain.
\end{abstract}

\section{Introduction}

The estimation of slope from a regularly-gridded DEM is a common procedure in terrain analysis. Some authors have evaluated the accuracy of various slope algorithms, using different assessment methodologies. The results of the slope estimations are always dependent on the generalization/resolution and quality of the DEM. Even though a number of studies, based on different types of "ground truth" data, have been presented, there are no reports with the aim to investigate possible differences between different slope algorithms and their sensitivity in different terrain. In this paper, eight frequently used slope algorithms are evaluated against each other. They are applied in three different terrain forms, namely flat, gently sloping/undulating, and steep terrain. The estimated slope 
results are statistically compared, and the result clearly indicates differences between the algorithms as well as how they behave in different terrain forms.

The aim of this paper is to dig the inherent difference of eight slope algorithms and their sensitivity to different terrain type. The purpose can be stated as follows:

(1) Reveal the estimated differences for eight slope algorithms, overestimated or underestimated comparing to each other;

(2) Combine two variables of algorithm and terrain, to see the distribution of estimated slopes, concentrated or spread and the sensitivity to terrain for each slope algorithm;

(3) Much more detailed characteristics of each algorithm and their natural differences existed among them are indicated.

\section{Background}

Applications of local topographic variables, such as slope and aspect have attracted considerable interests (Florinsky [4], Zhou and Liu [14]) and became important environmental variables in many models, not only limited to hydrological models but also ecological models (Skidmore [8]). Many popular slope calculation algorithms employed on DEMs have been used in GIS (Geography Information System) software (e.g. ARC/INFO, ERDAS IMAGINE). Given the importance of gradient/slope estimations in many applications, there is a strong demand to look for possible differences between the characteristics of frequently used algorithms and investigate how they behave in different terrain.

It is not unusual, probably mainly due to absence of knowledge regarding algorithm differences, that users neglect to select an appropriate slope algorithm, and are unaware of possible effects choosing different algorithms and apply them in different terrain forms. A number of researches have compared and evaluated different algorithms against "reference" values (e.g. calculated from mathematical surfaces or ground truth) (Florinsky [4]) but the results cannot sufficiently provide us with knowledge about differences between different algorithms, nor the terrain influence. In this study we have not based our comparisons on reference values, and the aim is not to find "better" or "worse" slope algorithms. Instead we try to highlight statistically significant difference between algorithms and investigate the algorithms" "response" in different terrain, namely flat, gently sloping/undulating, and steep terrain.

In the study, high resolution scattered LIDAR data were used to create the DEMs. Nearest neighbour interpolation and a grid size of 0.5 meters were used. Three different study areas, each 15 meters $\times 15$ meters (see figs 1-3), were chosen according to their dominating terrain form. The flat area (fig. 1) is showing smaller changes in elevation, the terrain in fig. 2 is characterized by gentle changes, classified as undulating, and the terrain in fig. 3 is dominated by larger differences in elevation, forming steep terrain. Even if it is only a case study, we let the different study areas represent different terrain conditions. The 


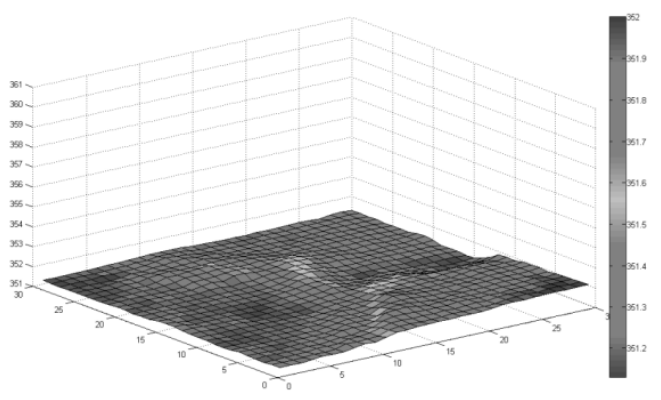

Figure 1: $\quad$ Flat DEM using 0.5 meters of grid on 30 by 30 area.

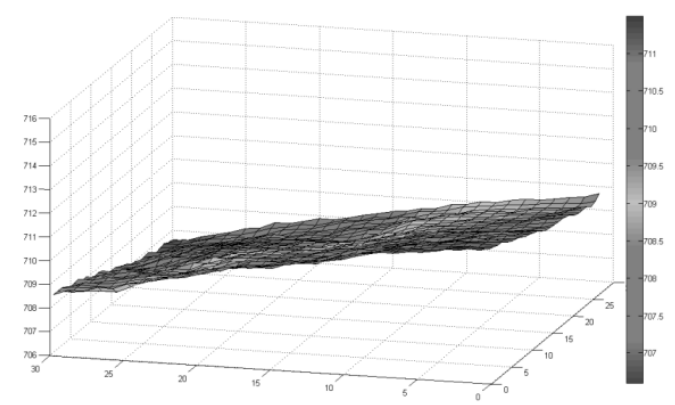

Figure 2: $\quad$ Undulating DEM using 0.5 meters of grid on 30 by 30 area.

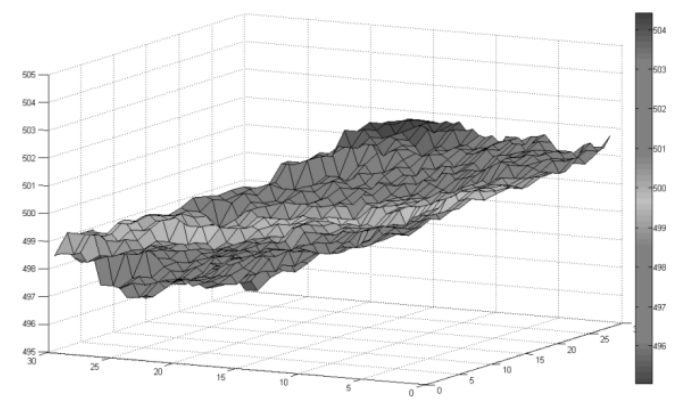

Figure 3: $\quad$ Steep DEM using 0.5 meters of grid on 30 by 30 area.

relationships between scale/resolution and the result of different DEM derivates estimated are relatively well understood, while we have decided to carry out our study with one single resolution. Also, keeping one resolution limits the complexity of the study, and we can by using the same cell size isolate 
differences caused by algorithm and/or terrain. The following pre-conditions are identified in order to strengthen the outcome of the study:

(1) The errors source of the three different DEMs is the same, since they are based on the same data set and interpolated in the same way;

(2) All slope values are estimated using a standard 3 by 3 moving window;

(3) The comparisons between the estimated values are processed in the same way independently on algorithm and/or terrain form.

\section{Slope algorithms}

At every point in a DEM the slope can be defined as a function of gradients in the $\mathrm{X}$ and $\mathrm{Y}$ direction:

$$
\text { Slope }=\arctan \sqrt{(f x)^{2}+(f y)^{2}}
$$

The key in slope estimation is the computation of the perpendicular gradients fx and fy. Different algorithms, using different techniques to calculate fx and fy yield the diversity in estimated slope. As mentioned above, from a gridded DEM, the common approach when estimating fx and fy is by using a moving $3 \times 3$ window to derive the finite differential or local polynomial surface fit for the calculation (Florinsky [4], Zhou and Liu [14]).

Below we have listed eight frequently used slope algorithms to be tested in this study. Methods 1-5 and 8 are convolutional methods, based on approximation of differential operators by finite differences (Ames [12]). Method 6 compares the central elevations with its eight neighbours, adopting the largest. Method 7 uses a quadratic regression surface constrained to go through the central elevation point of the local $3 \times 3$ DEM sampling kernel (Jones [6]). Before we briefly present the different methods we define the $3 \times 3$ window (fig. 4 ) and let the cell size (spatial resolution) equals to g (actually 0.5 meters).

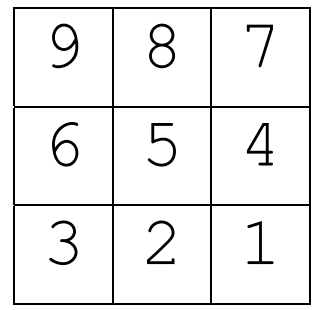

Figure 4: $\quad$ A $3 \times 3$ window with numbered cells.

In the following mathematical equations of slope, $z i \quad(i=1,2, \ldots 9)$ is the elevation value in cell $\mathrm{i}$ (defined in Fig. 4 above).

1) Second-order finite difference $2 F D$ (Fleming and Hoffer [3])

$$
\begin{aligned}
& f x=(z 6-z 4) / 2 g \\
& f y=(z 8-z 2) / 2 g
\end{aligned}
$$


2) Three-order Finite Difference Weighted by Reciprocal of Distance 3FDWRD (Unwin [11])

$$
\begin{aligned}
& f x=(z 3-z 1+\sqrt{ } 2(z 6-z 4)+z 9-z 7) /(4+2 \sqrt{ } 2) g \\
& f y=(z 7-z 1+\sqrt{ } 2(z 8-z 2)+z 9-z 3) /(4+2 \sqrt{ } 2) g
\end{aligned}
$$

3) Three-order Finite Difference, Linear regression plan 3FD (Sharpnack et al [7])

$$
\begin{aligned}
& f x=(z 3-z 1+z 6-z 4+z 9-z 7) / 6 g \\
& f y=(z 7-z 1+z 8-z 2+z 9-z 3) / 6 g
\end{aligned}
$$

4) Three-order Finite Difference Weighted by Reciprocal of Squared Distance 3FDWRSD (Horn [5])

$$
\begin{aligned}
& f x=(z 3-z 1+2(z 6-z 4)+z 9-z 7) / 8 g \\
& f y=(z 7-z 1+2(z 8-z 2)+z 9-z 3) / 8 g
\end{aligned}
$$

5) Frame Finite difference FFD (Chu and Tsai [9])

$$
\begin{aligned}
& f x=(z 3-z 1+z 9-z 7) / 4 g \\
& f y=(z 7-z 1+z 9-z 3) / 4 g
\end{aligned}
$$

6) Maximum Max (Travis et al. [10], EPPL7 [2])

$$
\begin{aligned}
& \max (\operatorname{abs}((\mathrm{z} 5-\mathrm{z} 1) /(\sqrt{ } 2 \times \mathrm{g})), \mathrm{abs}((\mathrm{z} 5-\mathrm{z} 2) / \mathrm{g}), \\
& \operatorname{abs}((\mathrm{z} 5-\mathrm{z} 3) /(\sqrt{ } 2 \times \mathrm{g})), \mathrm{abs}((\mathrm{z5}-\mathrm{z} 9) /(\sqrt{ } 2 \times \mathrm{g})), \\
& \operatorname{abs}((\mathrm{z} 5-\mathrm{z} 7) /(\sqrt{2} \times \mathrm{g})), \mathrm{abs}((\mathrm{z} 5-\mathrm{z6}) / \mathrm{g}), \\
& \operatorname{abs}((\mathrm{z} 5-\mathrm{z} 8) / \mathrm{g}), \text { abs }((\mathrm{z} 5-\mathrm{z} 4) / \mathrm{g}))
\end{aligned}
$$

7) Constrained Quadratic Surface Quadsurface (Wood [13])

$$
\begin{gathered}
\mathrm{F}(\mathrm{x}, \mathrm{y})=\mathrm{ax}^{2}+\mathrm{by}^{2}+\mathrm{cxy}+\mathrm{dx}+\mathrm{ey}+\mathrm{f} \\
\mathrm{AX}=\mathrm{Z}=\mathrm{F}(\mathrm{x}, \mathrm{y})
\end{gathered}
$$

where A has been defined (see fig. 5(4), $X$ stands for unknown vector of parameters (see fig.5(3) and $\mathrm{Z}$ is the elevation vector (see fig. 5(2)).

The number of equations is more than the unknown parameters, so there is no "true" solution. We use the least-squares method (see eqn (15)) to determine the indices of the constrained quadratic surface.

$$
A^{T} A X=A^{T} Z \quad X=\left(A^{T} A\right)^{-1} A^{T} Z
$$

It is then relatively easy to estimate the fx and fy values at the center of the $3 \times 3$ window.

$$
\begin{aligned}
& \text { fx } \mid x=0, y=0=d \\
& \text { fy } \mid x=0, y=0=e
\end{aligned}
$$

8) Simple difference Simple-D (Jones [6])

$$
\begin{aligned}
& \mathrm{fx}=(\mathrm{z} 5-\mathrm{z} 4) / \mathrm{g} \\
& \mathrm{fy}=(\mathrm{z} 5-\mathrm{z} 2) / \mathrm{g}
\end{aligned}
$$




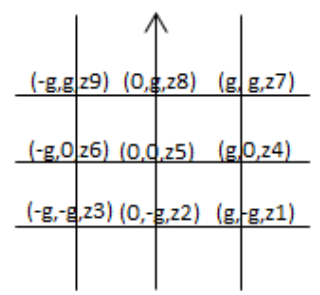

(1)

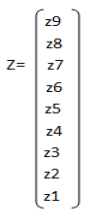

(2)

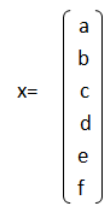

(3)

$$
A=\left\{\begin{array}{cccccc}
g^{\wedge} 2 & g^{\wedge} 2 & -g^{\wedge} 2 & -g & g & 1 \\
0 & g^{\wedge} 2 & 0 & 0 & g & 1 \\
g^{\wedge} 2 & g^{\wedge} 2 & g^{\wedge} 2 & g & g & 1 \\
g^{\wedge} 2 & 0 & 0 & -g & 0 & 1 \\
0 & 0 & 0 & 0 & 0 & 1 \\
g^{\wedge} 2 & 0 & 0 & g & 0 & 1 \\
g^{\wedge} 2 & g^{\wedge} 2 & g^{\wedge} 2 & -g & -g & 1 \\
0 & g^{\wedge} 2 & 0 & 0 & -g & 1 \\
g^{\wedge} 2 & g^{\wedge} 2 & g^{\wedge} 2 & g & -g & 1
\end{array}\right\}
$$

Figure 5: Constrained quadratic surface.

\section{Methodology}

In this study, we made our assessment of the different slope algorithms by pairwise comparison, and not by using "reference" or average values as the truth. In addition to that, we try to find out the influence of terrain on the different slope algorithms. Firstly we estimated slope values using the eight different algorithms in flat, undulating and steep terrain. Then selected statistical comparisons, from general tests to detailed paired comparison, were made on the estimated slope values.

To get the general differences between estimated slope values the descriptive variables mean and median, together with the $25^{\text {th }}$ and $75^{\text {th }}$ percentiles are adopted here (see Table 1 and fig. 6). In the plot of the mean and median values the estimation levels and distribution of the eight algorithms are well illustrated, and for the difference (span) between the $25^{\text {th }}$ and the $75^{\text {th }}$ percentile we get an illustration of the spreading within the dataset for the different algorithms and terrain forms.

After comparing the general characteristics the more detailed pair-wise statistical tests are implemented. Here, according to statistical theory, it is mandatory to test the homogeneity of variances before applying the paired comparison. If the test sig. (significance) is less than the expected value, the variances are unequal and vice versa. In our case the variances are significant and we choose one-way ANOVA for pairwise comparison. One-way ANOVA is suitable for a single factor with several observations at different levels. In our comparison, we view slope algorithm as the single factor affecting the slope value, so here we have eight observations at three levels: flat, undulating and steep terrain. 


\section{Results}

\subsection{Comparison of mean and median values}

Fig. 6 illustrates the mean and median slope values for eight algorithms within three levels of terrain. As shown in the figure, when the terrain is changing the trends for the eight methods are the same, regardless of which one of the mean and median that is used.

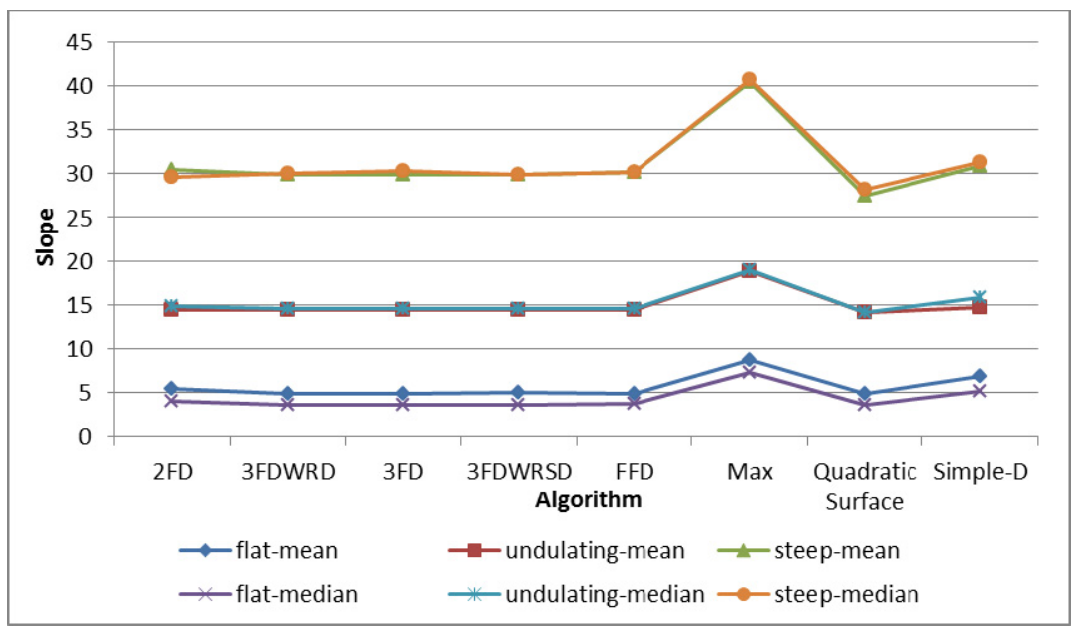

Figure 6: Differences in mean and median values for the different tested algorithms in the three terrain types. Mean value is describing the average estimated level, and median value is representing the 50 th percentile.

The mean and median value comparison provides the general analysis on the difference of slope estimation algorithms, i.e. if values are overestimated or underestimated compared to other algorithms. It is easy to distinguish the general characteristics of different algorithms. Visual interpretation of fig. 6 indicates that Max always shows the highest average value, considerably different to other methods, followed by Simple-D; while Quadratic Surface estimated values seem to be the lowest among all methods. The rest of algorithms show more or less same results. Generally, the mean and median values are reflecting the whole dataset, so the comparison of them extract broad, non-specific, information about the estimated slope values, reflecting larger differences between the tested algorithms.

We have chosen to study two parameters in our study, the algorithm and the terrain. One way to do this, focusing on the distribution of the estimated slopes is to compare the differences between the $75^{\text {th }}$ and $25^{\text {th }}$ percentile. Smaller values indicate concentrated distributions while larger values indicate more spread data. In Table 1 below the 75-25 percentile values are presented. If focusing on the 
influence of algorithm, independently of terrain type, we can conclude that the Simple-D method always generates higher values than the other algorithms. The Quadratic Surface method generally yields lower values than the other algorithms. When considering the effects of terrain for each method, the most sensitive method is Simple-D, and the second ranked is 2FD. The Quadratic method shows least sensitivity to terrain form.

Table 1: Differences between 75-25 percentiles for the different tested algorithms in the three terrain types.

\begin{tabular}{|c|c|c|c|c|c|c|c|c|}
\hline Algorithms & 2FD & 3FDWRD & 3FD & 3FDWRSD & FFD & Max & $\begin{array}{c}\text { Quad } \\
\text { Surface }\end{array}$ & Simple-D \\
\hline Flat & 5.40 & 4.71 & 4.65 & 4.76 & 4.78 & 5.84 & 4.62 & 6.00 \\
\hline Undulating & 6.87 & 5.76 & 5.78 & 5.85 & 5.85 & 7.74 & 5.45 & 11.31 \\
\hline Steep & 20.98 & 17.52 & 17.08 & 17.53 & 17.30 & 17.34 & 14.29 & 32.77 \\
\hline
\end{tabular}

\subsection{Equality tests between different algorithms}

Equality tests of different algorithms are used for more detailed comparisons of the algorithms. When running the test equal or unequal variances plays an important role and decides the analysis method. The results shown in Table 2 are the Levene Statistic and sig. value for flat, undulating and steep terrain. Those results, all with sig. values less than 0.05 , confirm unequal variances for the datasets within the different terrain types. The result presented in Table 1 made us use equality tests based on the premise of unequal variance.

Table 2: $\quad$ Test of Homogeneity of Variances in the same area.

\begin{tabular}{|c|c|c|}
\hline Areas & Levene Statistic & Sig. \\
\hline Flat & 17.320 & .000 \\
\hline Undulating & 113.411 & .000 \\
\hline Steep & 117.108 & .000 \\
\hline
\end{tabular}

\subsection{One-way ANOVA}

After the variance test presented above it was decided to run a test not relying on the assumption of equal variance. It was decided to choose the Dunnett $\mathrm{C}$ method. The goal of Dunnett $\mathrm{C}$ methods is to identify groups whose means are significantly different from the mean of a "reference group".

In this method, we first calculate the parameters $\mathrm{MS}_{\mathrm{B}}, \mathrm{MS}_{\mathrm{W}}$, and $\mathrm{F}$ from the test data. How this was done is described in eqn (20) to eqn (22) below.

$$
\mathrm{MS}_{\mathrm{B}}=\sum_{i} n_{i}\left(\bar{Y}_{i}-\bar{Y}\right)^{2} /(K-1)
$$

where $\bar{Y}_{i}$ denotes the mean value of each algorithm result, $\mathrm{n}_{\mathrm{i}}$ is the number of points of the calculated area, and $\bar{Y}$ denotes the overall mean value of eight algorithms in the same area. K equals 8 in our comparison. 


$$
\mathrm{MS}_{\mathrm{W}}=\sum_{i j}\left(Y_{i j}-\bar{Y}\right)^{2} /(N-K)
$$

where $Y_{i j}$ is the $\mathrm{j}^{\text {th }}$ point using the $\mathrm{i}^{\text {th }}$ algorithm. $\mathrm{N}$ is the overall point number in the same area.

$$
\mathrm{F}=\mathrm{MS}_{\mathrm{B}} / \mathrm{MS}_{\mathrm{W}}
$$

\begin{tabular}{|c|c|c|c|c|c|c|}
\hline \multirow{2}{*}{ Area } & \multicolumn{2}{|c|}{ Mean Square } & & & & \\
\hline & between groups $\left(\mathrm{MS}_{\mathrm{B}}\right)$ & within groups $\left(\mathrm{MS}_{\mathrm{W}}\right)$ & $\mathrm{MS}_{\mathrm{B}} / \mathrm{MS}_{\mathrm{W}}$ & Sig. & df1 & $\mathrm{df} 2$ \\
\hline Flat & 1528.491 & 23.170 & 65.969 & 0.000 & 7 & 6264 \\
\hline Undulating & 1973.829 & 26.308 & 75.028 & 0.000 & 7 & 6264 \\
\hline Steep & 11720.885 & 173.696 & 67.479 & 0.000 & 7 & 6264 \\
\hline
\end{tabular}

Table 3: $\quad$ ANOVA output.

The results of the analysis of variance are shown in Table 3 above. The between groups mean square value is the effects due to the different algorithms and the within groups value stands for the unsystematic variation in the data.

Comparing the mean square values between groups $\left(\mathrm{MS}_{\mathrm{B}}\right)$ in the three terrain types shows that the estimated slope values are fluctuating heavily in steep terrain. When combining the values of $\mathrm{MS}_{\mathrm{B}}$ and $\mathrm{MS}_{\mathrm{W}}$ we find that high fluctuations in the test area (high value of $\mathrm{MS}_{\mathrm{W}}$ ) seem to be linked to higher variations between different algorithms. The probability (labeled Sig. in Table 3) values of 0.000 and the high $F$ values strongly indicates that the results could not occur by chance, and there is strong evidence that the slope values calculated by the eight algorithms differ significantly, regardless of terrain form (flat, undulating or steep).

The Dunnett $C$ test can run all the pair-wise comparisons at one time. Table 4 lists the results, with $99 \%$ probability of statistical significance. For each pair comparison, the calculated results from Dunnett's method are compared to a critical value read from "Dunnett's Table", which depends on the group sizes, the number of groups chosen to be compared and the chosen significance level of the test.

Comparing the estimated slopes values based on different algorithms for every cell in the DEMs and then test the mean deviation between corresponding cells is more adequate then more general methods presented above, only comparing average slope values. The pair-wise comparison reflects the inside differences and details much better. Table 4 demonstrates the differences the slope algorithms in the three different terrain types. The methods listed in different groups confirm statistical significance between them.

In Table 4 we see that the Max method always yields slope values differing significantly from the other methods, no matter on terrain form. For the Simple$\mathrm{D}$ algorithm it is clearly shown that with the increase of gradient the estimated values get closer to the rest of methods, except for the Max algorithm. The 
Simple-D and the Max methods seem to overestimate slope compared to other methods, especially on flatter terrain. As indicated already in fig. 6, the Quadratic Surface algorithms yields lower slope estimations than other algorithm in steeper terrain.

Table 4: $\quad$ The statistic results of each pair (99\% probability).

\begin{tabular}{|c|c|c|c|c|c|c|c|c|}
\hline \multicolumn{3}{|c|}{ Flat Area } & \multicolumn{3}{c|}{ Undulating Area } & \multicolumn{3}{c|}{ Steep Area } \\
\hline $\begin{array}{c}\text { Group } \\
\text { I }\end{array}$ & $\begin{array}{c}\text { Group } \\
\text { II }\end{array}$ & $\begin{array}{c}\text { Group } \\
\text { III }\end{array}$ & $\begin{array}{c}\text { Group } \\
\text { I }\end{array}$ & $\begin{array}{c}\text { Group } \\
\text { II }\end{array}$ & $\begin{array}{c}\text { Group } \\
\text { III }\end{array}$ & $\begin{array}{c}\text { Group } \\
\text { I }\end{array}$ & $\begin{array}{c}\text { Group } \\
\text { II }\end{array}$ & $\begin{array}{c}\text { Group } \\
\text { III }\end{array}$ \\
\hline 2FD & & & 2FD & 2FD & & 2FD & & \\
\hline 3FDWRD & & & 3FDWRD & & & 3FDWRD & & \\
\hline 3FD & & & 3FD & & & 3FD & & \\
\hline 3FDWRSD & & 3FDWRSD & & & 3FDWRSD & & \\
\hline FFD & & & FFD & & & FFD & & \\
\hline $\begin{array}{c}\text { Quadratic } \\
\text { Surface }\end{array}$ & & & $\begin{array}{c}\text { Quadratic } \\
\text { Surface }\end{array}$ & & & $\begin{array}{c}\text { Simple } \\
\text {-D }\end{array}$ & & \\
\hline & $\begin{array}{c}\text { Simple- } \\
\text { D }\end{array}$ & & & $\begin{array}{c}\text { Simple- } \\
\text { D }\end{array}$ & & & $\begin{array}{c}\text { Quadratic } \\
\text { Surface }\end{array}$ & \\
\hline & & Max & & & Max & & & Max \\
\hline
\end{tabular}

\section{Conclusions}

Based on the analyses and results presented above we can extract the following conclusions:

(1) Regarding to the mean and median value of estimated slope values (see Fig. 6), the methods 2FD, 3FDWRD, 3FD, 3FDWRSD, FFD and Simple-D yield more or less equal values, maybe with Simple-D producing slightly higher slope values. These methods are all convolutional methods in the way that they calculate the two perpendicular partial derivatives of $\mathrm{fx}$ and fy. The differences in estimated slope seem to be less for flatter terrain. This can be explained by the variation in the terrain; smoother surfaces with lower slopes and less variation yield smaller differences in estimated slope. This is also supported by e.g. Carter [1].

(2) The Quadratic Surface method seems to underestimate slope compared to other algorithms, especially in steeper terrain where it shows statically difference compared to other methods. This algorithm uses a regression surface to go through the central elevation point of the local $3 \times 3$ sampling kernel. The first derivative of this fitted trend surface at the center point seems to estimate slope values that are relatively small.

(3) Regarding the variation of estimated slope values it seems like Simple$\mathrm{D}$ and 2FD yield larger variation in estimated slope for different terrain types than other algorithms. The other algorithms seem to be less influenced by terrain (see Table 1). For Simple-D and 2FD, the slope 
calculation from the adjacent cells, the nearest two or four points on the grid, are more likely to be influenced by the changing terrain.

(4) All eight algorithms show much greater differences between each other in steep terrain (see $\mathrm{MS}_{\mathrm{B}}$ in Table 3). In other words, algorithm choice becomes critically important when the terrain is fluctuating.

(5) The results shown in Table 4 indicates, no matter flat, undulating or steep terrain, that the estimated results from the Max method has statistically different results compared to other algorithms. For the Simple-D algorithm, when the terrain becomes less steep, the estimated slopes differ from other algorithm. However, the mean value is still close to the other estimates (see fig. 6). The Quadratic Surface algorithm seems to differ from the others in steeper terrain.

(6) Due to no actual "true" values of slope, the accuracy of different methods cannot be determined. However, since the slope estimation naturally is heavily scale/cell size dependent it is impossible to conclude which algorithm that is generally better than others. Different algorithms are most probably suitable for different applications on different scales.

\section{Discussion}

In this study we have chosen three different terrain types, namely flat, undulating and steep terrain, and made use of the same DEM construction methodology to get the same resolution of gridded DEM. We have then excluded effects of source errors. This has increased the possibilities to investigate differences between different slope algorithms applied in different terrain.

Several studies have been performed to try to find the "best" slope algorithm through estimating their accuracy based on reference data. With diverse testing methodologies, the results may be compatible or controversial. As we all know, it is impossible to conclude which method is better based on reference value. So in our study, the starting point on digging the inherent difference was not to evaluate the accuracy of algorithms, but differences between them. This is a new approach, and more detailed characteristics and differences have been studied.

Through the analysis results, we find that the Max method shows higher estimated results and significantly different results compared to other algorithms. For these reason it is not recommended to use this method unless special consideration to these findings is taken into account. For the Quadratic surface method, smoother surface (lower slope) will be the result due to larger numbers of surrounding grid cells included in the calculations. The rest of the algorithms, 2FD, 3FDWRD, 3FD, 3FDWRSD FFD and Simple-D, based on very similar theory calculating partial derivatives of $\mathrm{fx}$ and fy, show much closer results within the three kinds of terrains. Among these algorithms 2FD and Simple-D yield higher slope value due to less sampled grids when estimating slope. Additionally, the 2FD and Simple-D algorithms showed higher sensitivity to the terrain. For real world applications, much more effort and data have to be taken into consideration to choose an appropriate method to estimate slope values. Our 
results provide a clear indication of significant the differences between the eight tested slope algorithms, and it is strongly recommended to integrate field work and appropriate ground truth data in order to choose a proper slope estimation algorithm.

\section{Reference}

[1] Carter, J. R., The Effect Of Data Precision On The Calculation Of Slope And Aspect Using Gridded Dems. Cartographica: The International Journal for Geographic Information and Geovisualization 29(1), pp. 22-34, 1992.

[2] EPPL7, Environmental Planning and Programming Language. Environmental Planning and Programming Language Users Guide, 1987.

[3] Fleming, M. D., and Hoffer, R.M., Machine Processing of Landsat MSS Data and DMA Topographic Data for Forest Cover Type Mapping, 1979.

[4] Florinsky, I. V., Accuracy of local topographic variables derived from digital elevation models. International Journal of Geographical Information Science 12(1), pp. 47 - 62, 1997.

[5] Horn, B. K. P., Hill shading and the reflectance map. Proceedings of the IEEE 69(1), pp. 14-47, 1981.

[6] Jones, K. H. A comparison of algorithms used to compute hill slope as a property of the DEM. Computers \& Geosciences 24(4), pp. 315-323, 1998.

[7] Sharpnack, D., A. and Akin, G., An algorithm for computing slope and aspect from elevations. Photogrammetric Survey 35, pp. 247-248, 1969.

[8] Skidmore, A. K., A comparison of techniques for calculating gradient and aspect from a gridded digital elevation model. International Journal of Geographical Information Systems 3(4), pp. 323-334, 1989

[9] T.H. Chu and T.H. Tsai, Comparison of accuracy and algorithms of slope and aspect measures from DEM. Proceedings of the GIS AM/FM ASIA'95: Bangkok, I-1 to 11,1995

[10] Travis, M. R. E., Gary H., Iverson, Wayne D.; Johnson, Christine G., VIEWIT: computation of seen areas, slope, and aspect for land-use planning. USDA Forest Service and Range Experimental Technical Report PSW-11/1975, Pacific Southwest Forest and Range, Experimental Station, Berkeley, California, 1975.

[11] Unwin, D., Introductory Spatial Analysis, Methuen, London and New York, 1981.

[12] W.F. Ames. Numerical Methods for Partial Differential Equations, 1977.

[13] Wood, J. The geomorphological characterisation of digital elevation models, Department of geography, University of Leicester, 1996.

[14] Zhou, Q. M. and X. J. Liu. Analysis of errors of derived slope and aspect related to DEM data properties. Computers \& Geosciences 30(4): pp. 369378, 2004. 